\title{
A Quantisation of Cognitive Learning Process by Computer Graphics-Games: Towards More Efficient Learning Models
}

\author{
Ahmet Bahadir Orun1, Huseyin Seker², John Rose ${ }^{3}$, Armaghan Moemeni', Merih Fidan ${ }^{4}$ \\ ${ }^{1}$ School of Computer Science, De Montfort University, Leicester, UK \\ ${ }^{2}$ Department of Computer Science \& Digital Technologies, University of Northumbria, Newcastle, UK \\ ${ }^{3}$ School of Psychology, University of Birmingham, Birmingham, UK \\ ${ }^{4}$ Lifelong Learning Institution, University of Leicester, Leicester, UK \\ Email:aorun@dmu.ac.uk
}

Received 8 January 2016; accepted 23 January 2016; published 28 January 2016

Copyright (C) 2016 by authors and OALib.

This work is licensed under the Creative Commons Attribution International License (CC BY).

http://creativecommons.org/licenses/by/4.0/

(c) (i) Open Access

\begin{abstract}
With the latest developments in computer technologies and artificial intelligence $(A D)$ techniques, more opportunities of cognitive data acquisition and stimulation via game-based systems have become available for computer scientists and psychologists. This may lead to more efficient cognitive learning model developments to be used in different fields of cognitive psychology than in the past. The increasing popularity of computer games among a broad range of age groups leads scientists and experts to seek game domain solutions to cognitive based learning abnormalities, especially for younger age groups and children. One of the major advantages of computer graphics and using game-based techniques over the traditional face-to-face therapies is that individuals, especially children immerse in the game's virtual environment and consequently feel more open to share their cognitive behavioural characteristics naturally. The aim of this work is to investigate the effects of graphical agents on cognitive behaviours to generate more efficient cognitive models.
\end{abstract}

\section{Keywords}

Computer Graphics, Cognitive Learning, Bayesian-Networks

Subject Areas: Psychology

\section{Introduction}

Virtual computer technology is capable of generating virtual effects and stimuli [1] which may have no alterna-

How to cite this paper: Orun, A.B., Seker, H., Rose, J., Moemeni, A. and Fidan, M. (2016) A Quantisation of Cognitive Learning Process by Computer Graphics-Games: Towards More Efficient Learning Models. Open Access Library Journal, 3: e2329. http://dx.doi.org/10.4236/oalib.1102329 
tive in traditional methods of learning. In the digital multimedia domain the graphical environment can change instantly to meet the needs of cognitive learning difficulty treatment schedules or to ease the adverse effects of them. These state-of-art techniques are so flexible and adaptable; they can provide a very wide range of options and design capability to fit to each individual for specific personal cognitive treatments or development. The number of reported cases of learning difficulties among children and adults has been increasing in last few decades. The substantial portion of this population includes the younger age group whose interests and life style substantially overlap with the use of popular computer games and programs. This potential attracts researchers to deepen their research to support people with learning difficulties using this media [2]-[4]. According to Golan and Baron-Cohen [5] computer assisted intervention techniques lead to skill development in highly standardized, predictable and controlled environments, while simultaneously permitting an individual to work at his/her own ability level. Individuals who experience discomfort with unfamiliar social environment such as people with Autistic Spectrum Disorders may benefit from computerized intervention techniques [6]. In this paper we have investigated the possible impacts of computer graphics environment on cognitive learning processes which could be seen as the initial step to an efficient computer game development for improved cognitive learning models. This work initially aims to develop computer based tools to quantify the cognitive behaviours of individuals, which can then be applied to develop more efficient cognitive models. The work considered a quantisation process to estimate cognitive learning measures via a graphic test. Both tasks can be enhanced by utilizing computer graphics. The major advantage of using computer graphical tools for cognitive learning process is that, those who suffer from a lack of communication skills or feel discomfort with social environments [6] may benefit from this kind of graphical utility to develop their cognitive learning. This kind of graphic cognitive learning tool may give them an opportunity to develop low-level learning skills unconsciously [7]. The theory of two types of learning (procedural and declarative) was introduced and presented by several researchers [8]-[10]. This is based on the two-level architecture of the cognitive learning model. One of the partial characteristics of cognitive learning is that the user completes a specific task which could include using a software tool tailored for this purpose without any previous knowledge of the specific learning aims and as a result the individual should learn almost unconsciously. This may help to activate his/her skills by cognitive learning [11]. This is exploited in the graphical-based cognitive test presented in this paper which is assumed to help develop cognitive learning skills. By facilitating the performance of a broad range of cognitive learning, this may improve an individual's ability to communicate effectively [12]. The proposed method focuses on the use of computer graphics and maximises cognitive learning process. To date most computer-assisted learning facilities have been based on high level knowledge (e.g. program packages, internet, etc.) and do not effectively exploit the capacity of deeper level learning. This is because we can only measure, model and simulate the activities at a high level and we also use this level when we communicate to each other by talking, listening or writing. At low levels the mind functions at a more basic form; they are more flexible and more innovative but this cannot be easily measured, modelled and represented in present computational forms [13] [14]. To unveil the operational structures (e.g. cognitive learning) at this level of mind, it is necessary to use non-conventional communication systems and methods by which much deeper cognitive analyses can be made. To achieve this more fundamental approach models of “cognitive learning” need to be built (see Section 2.5). The proposed work is considered as an early step of developing cognitive learning model by using a specifically designed computer graphics test (COGNITO) which is also used for development of a quantisation model. The computer graphics developed within this work may later be embedded into a mission linked game to be used for cognitive development of people with learning difficulties.

\subsection{Previous Work}

This multi-disciplinary field of research including psychology, computer science and artificial intelligence techniques has attracted several researchers in the last decade to bring innovative solutions to the current issues of people with developmental disabilities. The most related study that addressed low-level cognitive learning [12] model is introduced by Sun et al. [11] which is described this work as bottom-up learning by the use of computer graphics. In the experiments the participants are not given detailed pre-information to accomplish the task using the graphics utility to encourage them to achieve such cognitive learning from the task. In that work the skill learning model called "Clarion" is included into computer graphics utility for cognitive training purposes which 
integrate connectionist, reinforcement and symbolic learning methods.

\subsubsection{Data Collection via Computer Graphics}

Data collection techniques in a graphical environment have been widely demonstrated in previous research. One of studies suggests utilizing a video sequences extracted from a computer game [15] to collect user's cognitive behavioural data exhibited during the game activities. The work uses game based graphics in motion for a limited number of participants for cognitive behavioural analysis other than cognitive learning actions. One of the related studies called "Concept map" was conducted by [16] which aims to analyse the organisation of mental representation by a cognitive data collection via computers. The tool was described as extremely useful by the developer because it provides a visual representation of the relationships between the mental concepts that may not be evident from raw data alone. The work exhibits similar concepts with our cognitive data collection method via computer games but has only a partial overlap with it rather than exploiting the computer games or inference techniques. Sun et al. [7] also used a computer graphic tool "a navigation game" to justify a skill learning model called Clarion which helps investigate the bottom-up learning process. The earlier version of such graphical data acquisition tool called "Minefield Navigation Task" was also introduced in Naval Research Lab by Gordon et al. [17].

\subsubsection{The Use of Computer Games for Cognitive Learning}

In last few decades with the utilisation of state-of-the-art computer facilities and rich interactive multimedia environments the popularity of computer video as well as console games has been increasing exponentially among almost all age groups. This situation especially encourages the experts of psychology and computer science to exploit specifically developed computer games to intervene individuals with learning difficulties [18]-[21]. Huntinger [1] makes an emphasis on the idea that these kinds of game technologies can provide visual stimuli which could be difficult to generate by old fashion traditional systems. According to Wright et al. [22] computer games can be used to capture attention of the children for monitoring them progressively. Prada and Paiva [23] conducted a research on the role of autonomous synthetic characters in computer games and investigate their potential to promote the social engagement of individuals in virtual environments. They believe these characters would function as the game agents where each one undertakes a specific role to stimulate the game player to complete a specific task. Goh et al. [20] also introduce a work about the connection between the computer game design and strategies for mental health treatments of children and adolescents. One of the recent studies proposes a motivational framework to asses goal directed behaviours of the characters for both players and non-players in a computer game environment which leads to exploring the opportunities of using a Player and Agent Personality Database (PAPD) based on the same motivational framework for the design of virtual agents with personality in the computer game domains [24]. Children who suffer from language learning impairments (LLI) can benefit from computer games. A specific computer game developed by Nagarajan et al. [25] was used for training the children with LLI to alter their characteristics of speech in a two-step process. During the training period, the children spent three half-days for 20 days playing these computer games and their language comprehension improved substantially.

Other researchers also has exploited the digital virtual environments for cognitive intervention such as Parsons et al. [26] who uses this sort of environment for social skill training for those who are diagnosed with Autistic disorder. In their tests the participants including those with Autistic disorder are tested on their communication, interaction and navigation abilities. Shane and Albert [27] supports the idea that the use of visual media based computer technology for those with Autism (ASD) would be beneficial due to their strong visual processing skills and predilection towards such media. Other research [28] is based on a multimedia computer game called emotional trainer which is used to teach those with Autistic Spectrum Disorders (ASD) to recognise and predict human emotional responses. The above works indicate that computer training programs could be used as an effective tool for cognitive learning developments.

\section{Methods and Materials}

This work suggests the development of a comparative quantitative measures for the assessment of cognitive learning rather than absolute measures. To achieve this, a specially designed Graphic Test (COGNITO) was used on 14 individuals at different age groups (without learning disability). In the test each graphic page includes two 
optional responding factors:

1) user-centred attitude (where the participant acts dominantly by his/her own perspective neglecting the game hero),

2) game-hero-centred attitude (participant acts through the game hero, putting him/herself in its role).

This "two-mode-option" design is inspired from the fundamental idea that one of the measures will distinguish between normal and Autistic individuals [29]. But at this stage the test is only used for cognitive learning behavioural analysis rather than any Autistic diagnosis assuming that game-hero-centred attitude is a key point which effects learning action even though the individual is not autistic. In the first option of response to the test, the participant takes a decision of self-centred thinking (scored as "1"). Whereas in second option he/she responds to test from game-hero's point of view (scored as "0"). From beginning to end of the test, he/she is expected to perform a cognitive learning action progressively by observing each sequential graphic answer page followed by each graphic test (inquiry) page shown in Figure 1.

\subsection{Data Acquisition Method}

Adequate and reliable data acquisition which should not cause any distraction or anxiety of the user depends on dedicated utilities and a friendly interactive environment. The commonly used traditional environments for data collection from the individuals/patients are labs, hospitals or clinics where the patients or participants (especially children) cannot feel comfortable or objective enough to provide reliable characteristic cognitive information. These factors would also have adverse effects on healthy cognitive learning activities. Due to these reasons, the collection of data is carried out by using computer graphics environment (COGNITO), which is a fifteen-page graphic test whose samples are shown in Figure 1. The communication with the potential participants and data collection were carried out by one the authors of this study who was also involved with the recruitment of participants for the test. The range of ages for the participants was 12 - 42. None of them declared any learning difficulties and all were computer users. No particular computer utility setting was chosen and all did the test in their own environment.

\subsection{Graphical Quantisation}

Since it is very difficult to find an absolute quantitative measure which is applicable to all other cases (e.g. to assess the degree of cognitive learning), the suggested method here rather makes a comparative analysis between the learning progresses of participants. Hence the comparative results only belong to the graphic test used in the experiments. The calculated degree of cognitive learning (CL) for each participant is rated between the worst case of CL $(a)$ and the ideal case of CL $(b)$ with regards to (continuous) progressive learning process. Here the two extreme cases are;

$$
a=\{0,1,0,1,0,1, \cdots\} \text { and } b=\{0,0, \cdots, 1,1\}
$$

The idea of worst case $(a)$ is based on a characteristic sign of learning disability (e.g. Autism) where the patient exhibits a repetitive behaviour in his/her daily life activities [30] [31]. In some cases of the experiment, the participants exhibit a "reverse" form of cognitive learning (a progression from 1 to 0 ) which may have several reasons such as lack of objectivity (if the test target is guessed in advance), diversity of personal characteristics, etc. In this case a reverse cognitive learning by the participant can be taken account positively and described as a reverse form of learning process. This sort of learning may easily be normalized by the reverse test format. Two index values are specified for the behavioural quantisation of progressive cognitive learning; normal progressive learning $\left(I_{\text {learning }}\right)$ and reverse progressive learning $\left(I_{\text {reverse }}\right)$

$$
\begin{gathered}
I_{\text {learning }}=\frac{\sum\left[\left(1-\frac{m}{n}\right)-i\right]^{2}}{k} \\
I_{\text {reverse }}=\frac{\sum\left[\left(\frac{m}{n}\right)-i\right]^{2}}{k}
\end{gathered}
$$

In Equations (1) and (2) the parameters below refer to the user's graphic response vector $V_{g}($ e.g. $0,1,1,0, \cdots, 0)$; 


\section{In each vector:}

$k=$ number of total transition between 0 and 1 in $V_{g}$ (e.g. $k=2$ for $V_{g}=\{0,0,1,1,1,0,0\}$

$n=$ total number of element in $V_{g}(n=15)$

$m=$ number of element in $V_{g}$

$i=$ Boolean value of m. element in $V_{g}$ (e.g. 0 or 1$)$

As a quantitative measure (derived from Formula (1), (2)) the "strait line fit" is used with some modification because most suitable simple model for a progressive cognitive learning is assumed to be a linear model (Figure 2).

In addition to Index measure of learning, Chi-square test and Correlation search methods are also used for Goodness-of-fit analysis for the quantisation of test results.

\subsection{Data Sources and Specifications}

The data set contains the Boolean values of participants' responses to the graphical test which may be described as progressive cognitive learning (PCL) graphical test. The test includes 15 graphical inquiry pages and each test page is followed by an answer page which is expected to lead a progressive cognitive learning action as the participant follows whole parts of test from beginning to end. The ideal learning form in the model is shown in Figure 2 (represented by strait line) and also one participant's PCL (adapted for comparison and shown by dashed line) produced from the graphic test results. In the figure an ideal PCL refers to the ideal case in Table 1 $\left(I_{\text {Learning }}=5.4\right)$. The test was used on 14 participants from different age groups.

\subsection{Graphic Cognitive Test (COGNIT0)}

COGNITO is a fifteen-page specially designed graphics-see samples shown in Figure 1. Computer graphics are
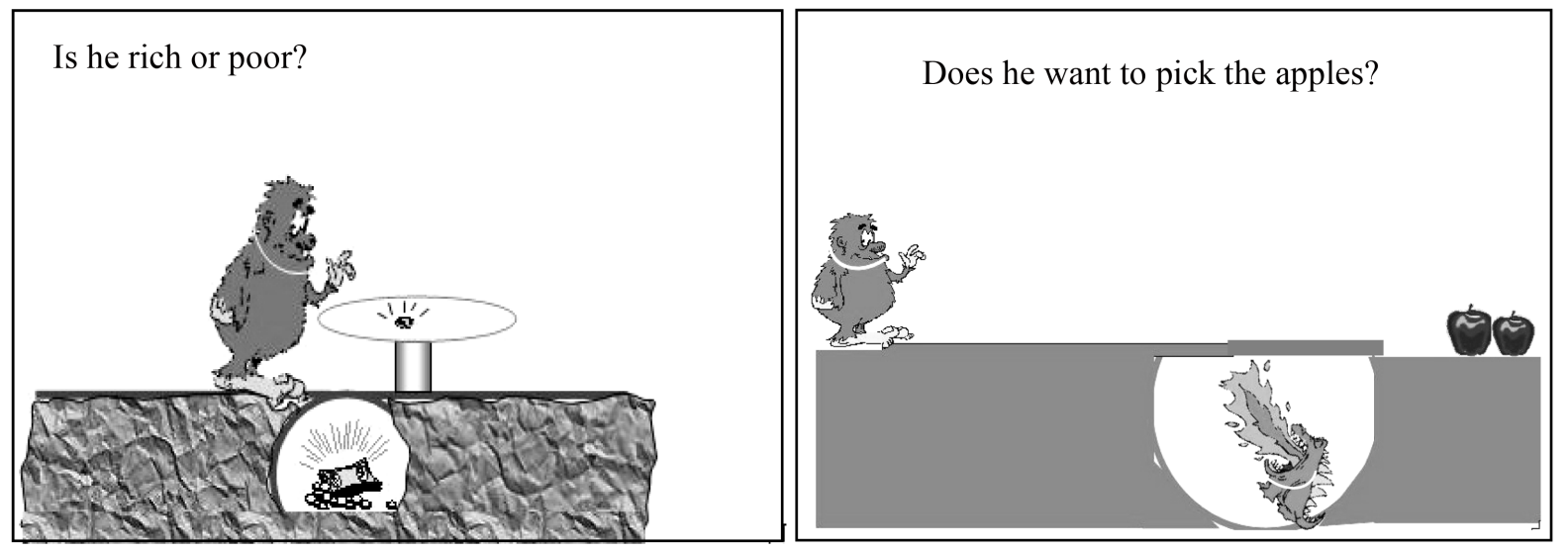

Figure 1. COGNITO: sample display of graphic agents used as a stimuli for cognitive learning process.

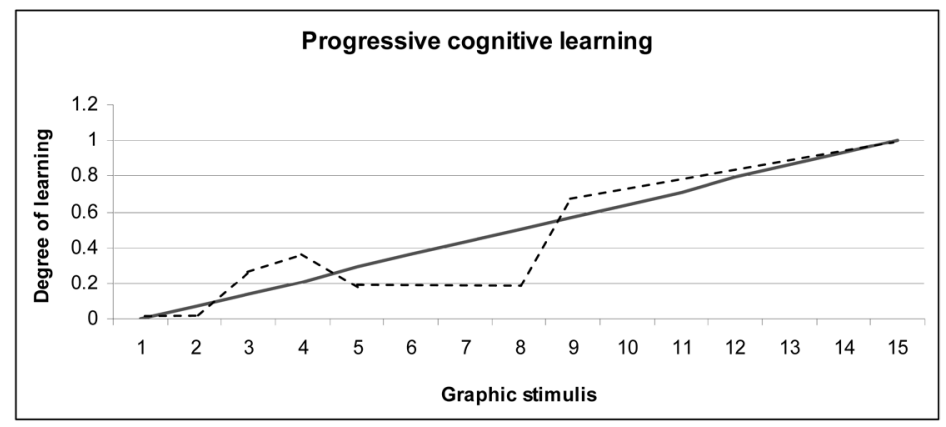

Figure 2. The strait line model of ideal progressive cognitive learning (PCL) and a participant's PCL (adapted for comparison and shown by dashed line) produced from the graphic test results Ideal PCL refers to the ideal case in Table 1 ( $I_{\text {Learning }}=$ 5.4). The line formula is $L=(\mathrm{m} / \mathrm{n})$. 
specifically designed so that the graphic agents (characters) in the test domain are supposed to be in interaction with the user and stimulate him/her to make his/her cognitive options to decode his/her cognitive learning characteristics. The tool COGNITO is used for cognitive learning purposes in the system. When the user receives the correct answer promptly after his/her reaction to stimuli (in this case the answer is "yes") then he/she is forced to learn the cause-effect relationships in computer environment. A further versions of the test may contain hundreds of computer graphics and each one may be used only once for each participant. In the more developed version, this convergence method of progressive learning would be particularly inevitable to minimize any learning disability progressively. Figure 1 exhibits two example graphic pages of the test in which two different characters are used (scenes are presented in colour) to capture user's characteristic options.

\subsection{Cognitive Learning at Broad Range}

The cognitive graphical test (COGNITO) is specifically designed and used in experiments so that it is expected to stimulate cognitive learning at broad range. The related arguments to support this idea are as follows;

1) The test was completed by the participants without any pre-knowledge. This helped produce low level knowledge during the test session. This assumption is based on previous studies [32] [33]. According to Sun et al. "when the participants are not provided a sufficient amount of a priori knowledge relevant to a task, learning proceed differently. It is likely that some skills develop prior to the cognitive learning by high level knowledge (bottom-up learning)".

2) Each case in the test is represented by a one-page graphics where each one is unrelated to the others to avoid producing obvious cause-effect relationships by the participants to avoid a high level knowledge, hence encourages low level one. According to several researchers [34]-[36] low level knowledge is not always preceded by high level one in learning. They are not necessarily correlated and rely to each others. This indicates that low-level cognitive learning is possible without a high-level learning action.

3) As it was commented by the participants that they found the test very illogical and meaningless. This lack of information about the aim of test should lead to a production of intrinsic knowledge via low level cognitive learning. Because the participants only learn to be self-centred without any other high level knowledge. This matches the description of low level learning by which the knowledge is gained unconsciously without awareness [18]. Hence at the end of the test the participants are possibly unaware of that they learn to think in a self-centred way.

According to Sun et al. [33] the low level cognitive learning can be possible by strait back-propagation (supervised learning) where correct input/output is provided. This method is followed in our graphic test by prompt graphic answer page after each question page. Even though many similar graphical methods have been studied so far, the test introduced here is more specifically "game-hero" centered.

\section{Results and Discussion}

\subsection{Quantisation of Progressive Cognitive Learning Action}

The proposed model introduces the idea of progressive cognitive learning quantisation which is a comparative work whose experimental results are presented within the range between worst and ideal learning cases and distributed equally. The graphical test used for cognitive learning has been specifically designed to support the idea that the participants progressively learn to think in self-centred way by reinforcement. The ideal progressive learning model (Figure 2) shown as strait line in the range between 0 and 1.

In Table 1 different measures of best-fit such as Index values (I), derived from the formulas 1 and 2, shown in grey shading, goodness-of-fit and correlation were applied to find the degree of each individual's cognitive learning. This was done by matching each learning case (id) to ideal PCL (in Figure 2). As is seen in Table 1 the measure I indicates the forward and reverse learning separately whereas Correlation measure is suitable to see the degree of progressive learning (+values) and reverse learning (-values) on the same row. Learning index (I) is most suitable measure for a comparison between the cases. Whereas Goodness-of-fit (chi-square test) values fall in the range between 12.2 and 15 and not very suitable to represent the worst case (12.7) which corresponds to repetitive patterned learning $(0,1,0, \cdots)$ in our proposed model. Learning case of Participant 1 (id1) is presented by Figure 3. 
Table 1. Index values (I) of progressive cognitive learning (PCL) and reverse PCL derived by Formulas (1) and (2) and other best-fit measure values of goodness-of-fit and correlation for participants' results $\left(\mathrm{id}_{\mathrm{i}}\right.$ ) ideal case (Figure 2 ) and worst case (repetitive 0, 1 patterns) are also shown at first and last column. In correlation section minus values indicate the reverse learning.

\begin{tabular}{|c|c|c|c|c|c|c|c|c|c|c|c|c|c|c|c|c|}
\hline Learning & Worst Case & Id1 & Id2 & Id3 & Id 4 & Id5 & Id6 & Id7 & Id8 & Id9 & Id 10 & Id11 & Id 12 & Id13 & Id14 & $\begin{array}{l}\text { Ideal } \\
\text { Case }\end{array}$ \\
\hline$I_{\text {Learning }}$ & 0.35 & 2.5 & 0.5 & 1.3 & 0.8 & 1.4 & 0.6 & 0.7 & 1 & 3.1 & 2.9 & 0.5 & 0.6 & 0.6 & 0.5 & 5.4 \\
\hline$I_{\text {Reverse }}$ & 0.35 & 0.8 & 0.9 & 0.7 & 0.6 & 0.6 & 0.7 & 1 & 1.5 & 1.9 & 2.1 & 0.6 & 0.9 & 0.6 & 0.8 & - \\
\hline $\begin{array}{c}\text { Total } \\
\text { transition (k) }\end{array}$ & 14 & 3 & 7 & 5 & 7 & 5 & 8 & 6 & 4 & 2 & 2 & 9 & 7 & 8 & 7 & 1 \\
\hline Correlation & -0.0002 & 0.6 & -0.2 & 0.5 & 0.2 & 0.4 & -0.1 & -0.3 & -0.2 & 0.4 & 0.1 & -0.1 & -0.2 & 0.07 & -0.3 & 0.9 \\
\hline $\begin{array}{c}\text { Chi-square } \\
\text { Test }\end{array}$ & 12.7 & 14 & 12.8 & 14.8 & 14 & 14.3 & 13.5 & 12.2 & 13.6 & 13 & 12.6 & 12.7 & 13.3 & 14 & 12.7 & 15 \\
\hline
\end{tabular}

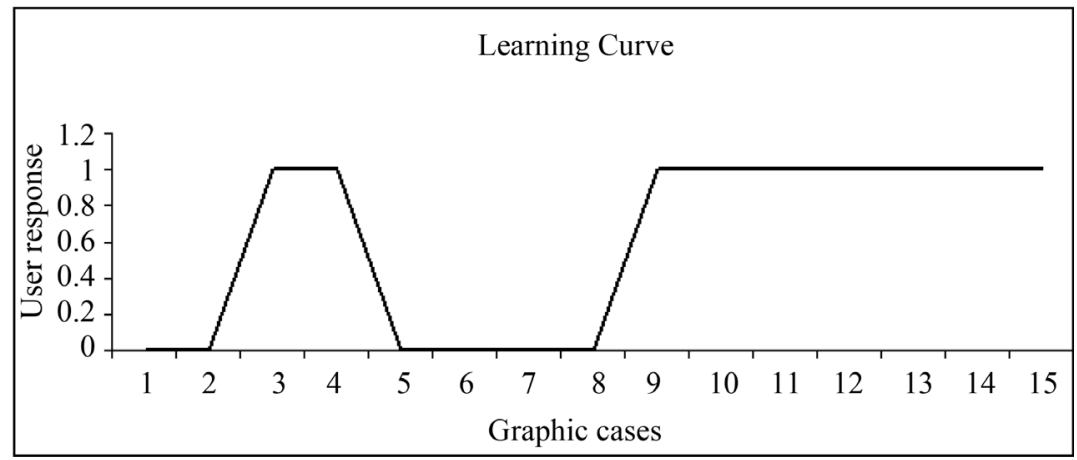

Figure 3. A sample case of cognitive learning graph for participant 1 ( $\left.I_{\text {learning }}=2.5\right)$ which exhibits the graphical test output and then matched to ideal PCL to quantise degree of learning.

\subsection{Data Analysis by Bayesian Networks}

The Bayesian network approach for modelling cognitive processes was introduced in [37]. In his work four of the most distinguished potential hierarchical Bayesian contributions to cognitive modelling were comprehensively discussed. Some of the previous works exhibit the different application fields of Bayesian inference method and classification process separately which provide a useful guidance for this work [38] [39]. In this work two different experiments are done by use of Bayesian network tool (Power Predictor ${ }^{\mathrm{TM}}$ ) for the analysis of data produced by the cognitive learning graphic tests. In general terms, Bayesian networks are called Casual Probabilistic Networks and very useful instrument which achieves a knowledge representation and reasoning. They are also capable of generating very accurate classification results under uncertainty where the data set includes many uncertain conditions [40]. The Bayesian networks are the probabilistic models which graphically encode and represent the conditional independence (CI) relationships among a set of data [38]. In the experiment (Figure 7), a learning Bayesian network software tool (Power Predictor) is used for classification purposes.

The Bayesian networks are the probabilistic models which graphically encode and represent the conditional independence (CI) relationships among a set of data. In this work, a learning Bayesian network software tool (Power Constructor ${ }^{\mathrm{TM}}$ ) is also used for the analysis of cognitive data and the inference to construct the network [33] which is a different tool than Power Predictor. Both utilities use the Markov condition to obtain a collection of conditional independence statements from the networks [41]. The algorithm examines information flow between two highly related variables (attributes) from a data set and decides if these variables (e.g., participants responses, test graphics, etc.) are independent or linked and it also investigates into how close the relationship between those variables is (Figures 4-6).

\subsection{Experiments for Classification}

1) Classification of learning levels (high/low) by Bayesian network 


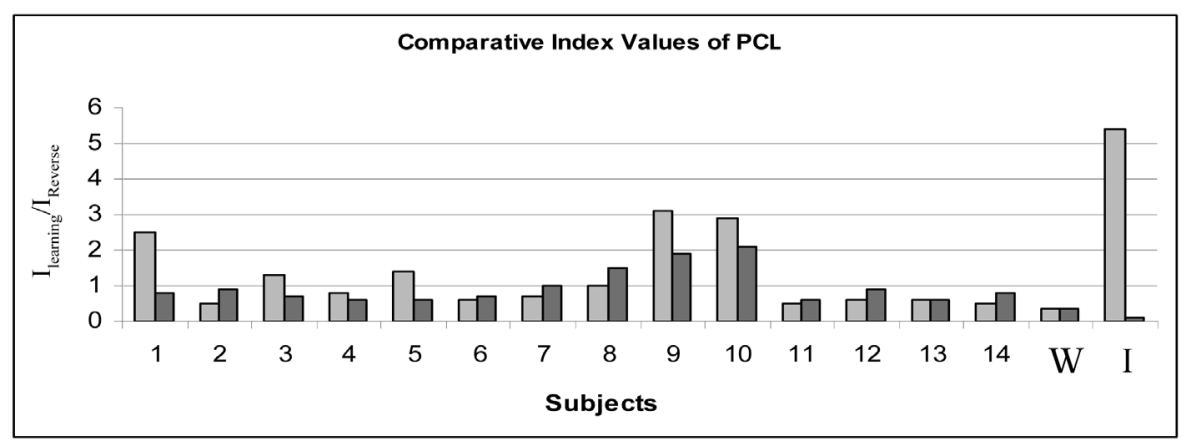

Figure 4. The comparison between the index values of progressive cognitive learning (grey bars) and reverse progressive cognitive learning (black bars) is shown ( $\mathrm{W}=$ worst case of PCL, and I = ideal case of PCL).

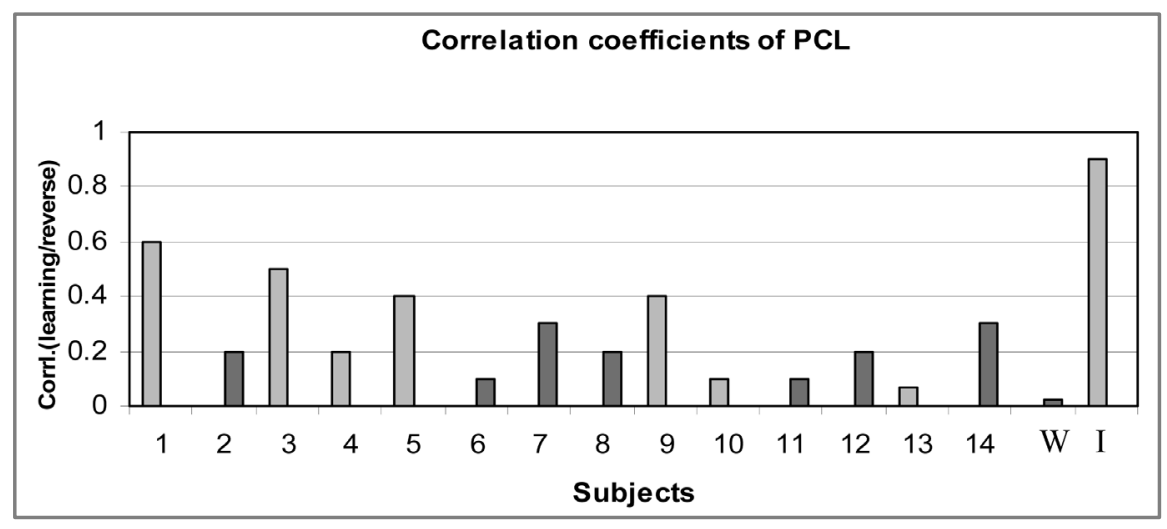

Figure 5. The comparison between the correlation coefficients of progressive cognitive learning (grey bars) and reverse progressive cognitive learning (black bars) for each participant is shown (W = worst case of PCL, and I = ideal case of PCL).

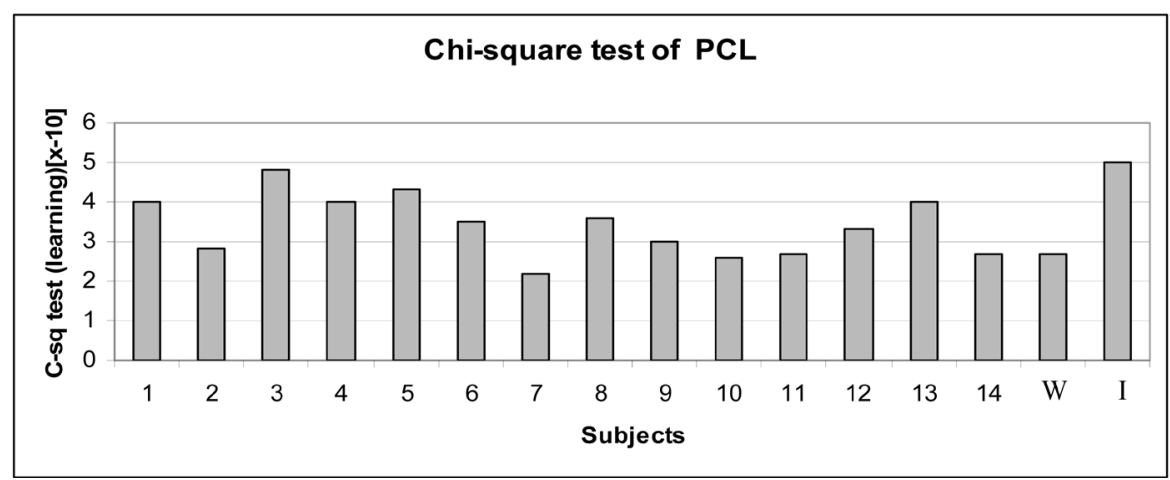

Figure 6. The comparison between the Chi-square test values of progressive cognitive learning (PCL) for each participant is shown (W = worst case of PCL and I = ideal case of PCL. The values are scaled as $\mathrm{X}-10$ for a better display).

The aim of this experiment is to investigate what types of graphical attributes with regards to the different levels of learning groups (high/low) may play role in cognitive learning process. This information will play a substantial role in optimum configuration of the further versions of graphic tools tailored for the learning actions of different specific groups (educational level, family background, IQ levels, etc.). With regards to the hypothesis used within this proposed system, a progressive cognitive learning to be accomplished by each participant by following the graphical test from beginning to end. The ideal progressive learning action is represented by a class node "learning", a vector which contains values starting "0s" in its first half (representing low-level learn- 
ing) and "1s" in its second half (represents high-level learning). By the classification process, these two groups of learning action were classified with $75 \%$ accuracy by using Bayesian classifier (Power Predictor ${ }^{\mathrm{TM}}$ ) as seen in Figure 7. In the classification the attributes 3, 6, 10, 12, 13 and 14 played role to separate between two levels of learning. The data set as is shown by Table 2 used for training/test of Bayesian network by the ratio of 8/7, by which the classification of learning levels (high/low) of ideal progress was carried out. In this experiment the dataset contains the attributes of 15 (graphics) $\times 14$ (participants) to classify two categories of learning groups (high/low) is used with Bayesian network tool (Power Predictor ${ }^{\mathrm{TM}}$ ). Some of the specific optional parameters are chosen such as the threshold is selected (automatically) between 0.1 - 50, ROC (under Curve) and equal discretization method are selected, etc. in the network configuration.

As is seen in Figure 7 the network combination of nodes 3, 6, 10, 12, 13 and 14 are used for separation between the two classes (high/low level learners). The other nodes being disconnected are not necessarily unimportant but their inclusion would not improve the classification accuracy. The class node (ID) includes the type of category. In the network the direction of arcs are disregarded. This is because it is well known that in a Bayesian network different configurations of directions of arcs may induce the same independencies [42]. In the configurations, direction of the arcs is not uniquely determined. It can therefore not be expected that the arcs actually reflect the direction of causality. However, they can help show the connections between the related attributes.

2) Classification of user groups influenced from the graphical properties

The goal of this task is to discover the impact of specific graphical properties in the test which may play a significant roles to separate two kinds of users groups (e.g. regarding their age, educational level, life style, etc.) and hence to classify users' cognitive learning characteristics. The output may later be used to help take decisions about an effective education or treatment method for learning disabilities of those different categories. For this experiment $1^{\text {st }}, 7^{\text {th }}$ and $9^{\text {th }}$ test pages (used as graphical attributes) are selected automatically by Bayesian inference methods (by Power Constructor ${ }^{\mathrm{TM}}$ tool) which are more effective to separate two groups (adults/ teenagers) than the other pages (graphical attributes) as is seen in Figure 8. By the experiment each participant user interacts with all graphical properties along the test which causes cognitive learning process specific to his/her cognitive characteristics. In the test a reverse form of data set (shown in Table 2) is used, where the

Table 2. Reduced form of data set used for training/test of Bayesian network by separation at the ratio of 8/7 by which the classification of the levels of ideal progressive learning (high $=1$, low $=0$ ) of the ideal was carried out.

\begin{tabular}{|c|c|c|c|c|c|c|c|c|c|c|c|}
\hline Participants/Graphic cases & Id1 & Id2 & - & . & - & . & . & . & - & . id14 & $\begin{array}{c}\text { Level of ideal progressive } \\
\text { learning }\end{array}$ \\
\hline 1 & 0 & 1 & & . & . & . & . & . & . & 1 & 0 \\
\hline 2 & 0 & 0 & & . & . & . & . & . & . & 0 & 0 \\
\hline 3 & 1 & 1 & & . & . & . & . & . & . & 0 & 0 \\
\hline . & . & . & & . & . & . & . & . & . & . & . \\
\hline . & . & . & & . & . & . & . & . & . & . & 1 \\
\hline 15 & 1 & 0 & & . & . & . & . & . & . & 0 & 1 \\
\hline
\end{tabular}

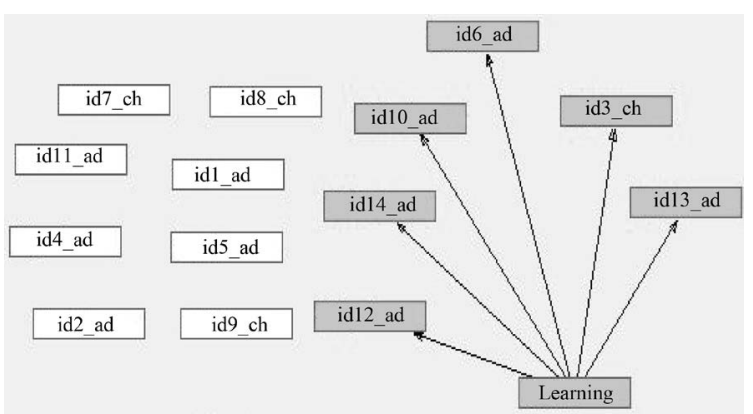

Figure 7. The Bayesian network representation of the attributes used to classify the low and high learning categories. Ideal progressive learning action refers to the class node "learning" within the network and classification of categories (high/low) is done via attributes 3, 6, 10,12, 13 and 14 which correspond to participants' responds to graphic test. Classification accuracy reached is $75 \%$ when test/train data set ratio is chosen as $8 / 7$. 


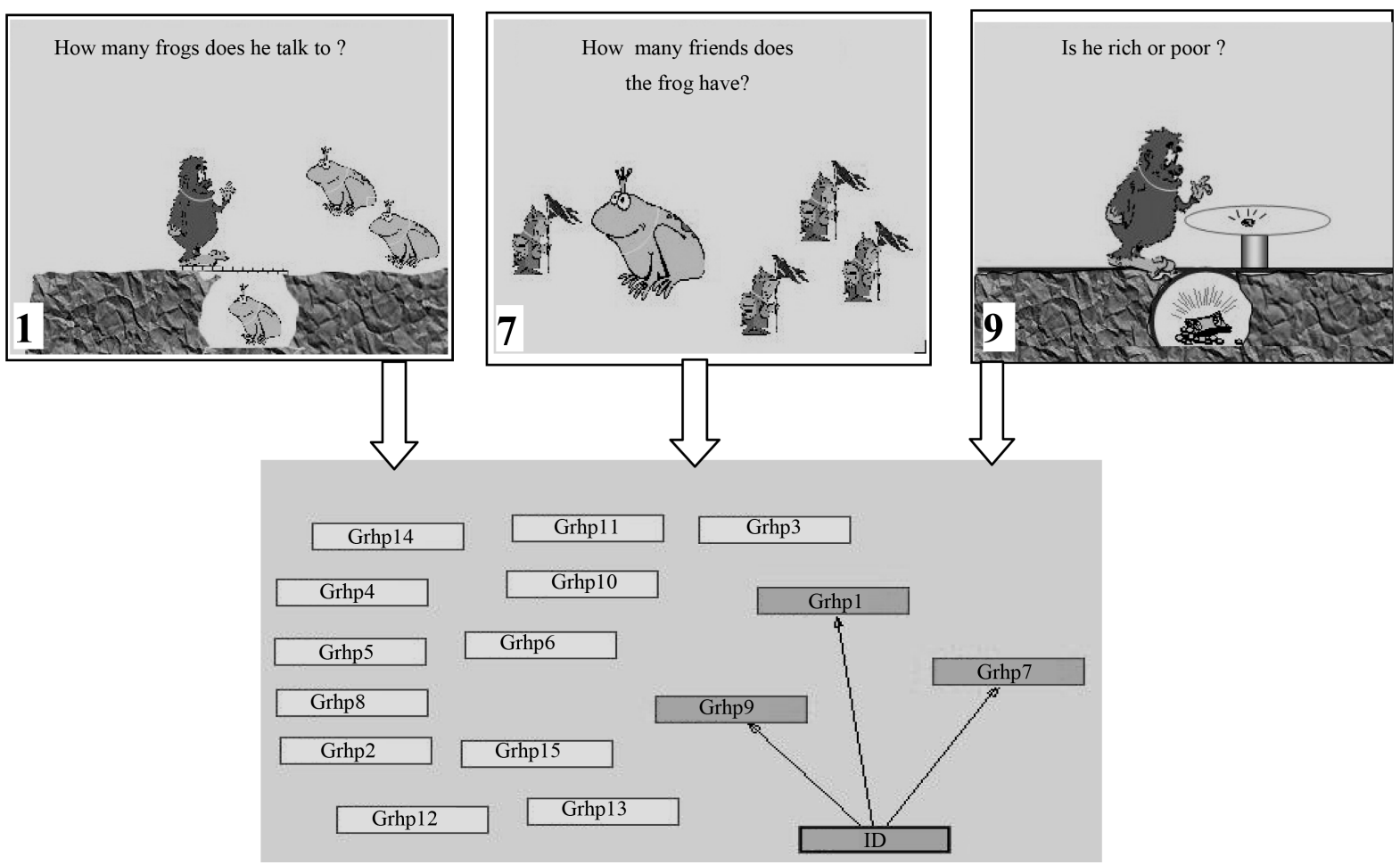

Figure 8. The attribute selection result of Bayesian inference method where each attribute refers to graphical test page. Test pages 1, 7 and 9 are selected which are effective to separate two group of users.

attributes are graphical test pages, the cases are participant individuals and class node also refers to type of individuals (e.g. adult/teenager).

These tasks are to demonstrate a basic level of quantisation by using Bayesian classification process (e.g. low/ high learning, low/high graphical impact on learning, etc.). It is also possible to increase the number of classes by use of multi-net option of Bayesian classifier (with a larger data set) for higher level of quantisation.

\section{Conclusion and Future Works}

The data collected from the test participants for the proposed cognitive learning model are used for the comparative work for a group of individuals to quantify differences between the cases rather than suggesting absolute quantitative measures applicable to all other similar cases. Therefore the size of data set used here is not very important and only used to justify the functionality of this proposed quantitative model. The experiment (Figure 7) shows that the hypothesis of progressive cognitive learning of an individual (participant) by the graphical test is proven with $75 \%$ accuracy by using the Bayesian Network classifier. The further developed version of suggested model would be used for modelling of learning difficulties or decreasing the effects of them. On the basis of research results which suggest that around half of people with Autism (ADS) may also have a learning disability [43], in further version of work we also would pay more attention on Autism as well as learning disability to diminish its adverse effects. One of the ADS characteristics is the lack of social communication with the other individuals. In addition to that the ADS individual also ignores what the others think and feel about him/her. For the intervention or supportive skill development which helps avoid these abnormal behavioural effects, more specific graphic-based utilities and rich interactive media GUI may be used. First of all, the differences of cognitive learning characteristics between the two groups of individuals (normal/abnormal) have to be detected. In the second stage these characteristics are embedded in the computer game or graphics agents where each one plays a functional stimulant role in the game or immersive graphical environment by forcing the individual to behave cognitively in opposite direction. Briefly at the intervention stage the behavioural data of normal group whose feedbacks are acquired via game-graphical environment, might be used to adjust the system parameters which then are used to influence the behaviours of abnormal group. This could progressively lead to cognitive 
normalisation of those from abnormal group by a convergence process. More sophisticated design of the proposed system may also consist of a complete console/video game which includes the game agents functioning in the same way with this graphical cognitive test (COGNITO) while the player is interacting with a rich interactive game environment. Identifying more realistic and well defined attributes directly driven from users' interaction in the virtual environment will enhance the outcome of the cognitive analysis using suggested Bayesian classification process in this paper's framework.

\section{References}

[1] Huntinger, P.L. (1996) Computer Applications in Programs for Young Children with Disabilities: Recurring Themes. Focus on Autism and Other Developmental Disabilities, 11, 105-114. http://dx.doi.org/10.1177/108835769601100206

[2] Wainer, A.L. and Ingersoll, B.R. (2011) The Use of Innovative Computer Technology for Teaching Social Communication to Individuals with Autism Spectrum Disorders. Research in Autism Spectrum Disorders, 5, 96-107. http://dx.doi.org/10.1016/j.rasd.2010.08.002

[3] Mitchell, P., Parsons, S. and Leonard, A. (2007) Using Virtual Environments for Teaching Social Understanding to 6 Adolescents with Autistic Spectrum Disorders. Journal of Autism and Developmental Disorders, 37, 589-600. http://dx.doi.org/10.1007/s10803-006-0189-8

[4] Heimann, M., Nelson, K.E., Tjus, T. and Gillberg, C. (1995) Increasing Reading and Communication Skills in Children with Autism through an Interactive Multimedia Computer Program. Journal of Autism and Developmental Disorders, 25, 459-479. http://dx.doi.org/10.1007/BF02178294

[5] Golan, O. and Baron-Cohen, S. (2006) Systemizing Empathy: Teaching Adults with Asperger Syndrome or HighFunctioning Autism to Recognize Complex Emotions Using Interactive Multimedia. Developmental and Psychopathology, 18, 591-617. http://dx.doi.org/10.1017/s0954579406060305

[6] Charlop-Christy, M.H., Le, L. and Freeman, K.A. (2000) A Comparison of Video Modelling with in Vivo Modelling for Teaching Children with Autism. Journal of Autism and Developmental Disorders, 30, 537-552. http://dx.doi.org/10.1023/A:1005635326276

[7] Sun, R., Merrill, E. and Peterson, T. (2001) From Implicit Skills to Explicit Knowledge: A Bottom-Up Model of Skill Learning. Cognitive Science, 25, 203-244. http://dx.doi.org/10.1207/s15516709cog2502_2

[8] Sun, R. and Bookman, L. (1994) Computational Architectures Integrating Neural and Symbolic Processes. Kluwer Academic Publishers, Norwell. http://dx.doi.org/10.1007/b102608

[9] Sun, R. (1995) Robust Reasoning: Integrating Rule-Based and Similarity-Based Reasoning. Artificial Intelligence, 75, 241-296. http://dx.doi.org/10.1016/0004-3702(94)00028-Y

[10] Anderson, J. (1983) The Architecture of Cognition. Harvard University Press, Cambridge.

[11] Sun, R., Peterson, T. and Merrill, E. (1996) Bottom-Up Skill Learning in Reactive Sequential Decision Tasks. Proceedings of 18th Cognitive Science Society Conference, Hillsdale, 684-690.

[12] Lindsey, M. (2002) Comprehensive Health Care Services for People with Learning Disabilities. Advances in Psychiatric Treatment, 8, 138-147. http://dx.doi.org/10.1192/apt.8.2.138

[13] Goschke, T. (1996) Lernen und Gedächtnis: Mentale Prozesse und Gehirnstrukturen. In: Roth, G. and Prinz, W., Hrsg., Kopf-Arbeit: Gehirnfunktionen und kognitive Leistungen, Spektrum Akademischer Verlag, Heidelberg, 359-410.

[14] Lamberts, K. and Shanks, D. (1997) Knowledge, Concepts and Categories. Psychology Press, Sussex.

[15] Orun, A.B. and Seker, H. (2012) Development of a Computer Game-Based Framework for Cognitive Behaviour Identification by Using Bayesian Inference Methods. Computers in Human Behavior, 28, 1332-1341. http://dx.doi.org/10.1016/j.chb.2012.02.017

[16] Hoeft, R.M., Jentsch, F.G., Harper, M.E., Evans III, A.W., Bowers, C.A. and Salas, E. (2003) TPL-KATS-Concept Map: A Computerized Knowledge Assessment Tool. Computers in Human Behaviour, 19, 653-657. http://dx.doi.org/10.1016/S0747-5632(03)00043-8

[17] Gordon, D., Schultz, A., Grefenstette, J., Ballas, J. and Perez, M. (1994) User’s Guide to the Navigation and Collision Avoidance Task. Naval Research Lab, Washington DC.

[18] Durkin, K. and Barber, B. (2002) Not So Doomed: Computer Game Play and Positive Adolescent Development. Applied Developmental Psychology, 23, 373-392. http://dx.doi.org/10.1016/S0193-3973(02)00124-7

[19] Fleming, M.J. and Rickwood, D.J. (2001) Effects of Violent versus Nonviolent Video Games on Children’s Arousal, Aggressive Mood, and Positive Mood. Journal of Applied Social Psychology, 31, 2047-2071. http://dx.doi.org/10.1111/j.1559-1816.2001.tb00163.x

[20] Goh, D.H., Ang, R.P. and Tan, H.C. (2008) Strategies for Designing Effective Psychotherapeutic Gaming Interventions 
for Children and Adolescents. Computers in Human Behavior, 24, 2217-2235.

http://dx.doi.org/10.1016/j.chb.2007.10.007

[21] Griffiths, M.D., Davis, M.N.O. and Chappell, D. (2003) Breaking the Stereotype: The Case of Online Gaming. Cyberpsychology \& Behavior, 6, 81-91. http://dx.doi.org/10.1089/109493103321167992

[22] Wright, J.H., Wright, A.S., Albano, A.M., Basco, M.R., Goldsmith, L.J. and Raffield, T. (2005) Computer-Assisted Cognitive Therapy for Depression: Maintaining Efficacy While Reducing Therapist Time. American Journal of Psychiatry, 162, 1158-1164. http://dx.doi.org/10.1176/appi.ajp.162.6.1158

[23] Prada, R. and Paiva, A. (2009) Teaming up Humans with Autonomous Synthetic Characters. Artificial Intelligence, 173, 80-103. http://dx.doi.org/10.1016/j.artint.2008.08.006

[24] Bostan, B. (2010) A Motivational Framework for Analysing Player and Virtual Agent Behaviour. Entertainment Computing, 1, 139-146. http://dx.doi.org/10.1016/j.entcom.2010.09.002

[25] Nagarajan, S.S., Wang, X., Merzenich, M.M., Schreiner, C.E., Johnston, P., Jenkins, P., et al. (1998) Speech Modifications Algorithms Used for Training Language Learning-Impaired Children. IEEE Transactions on Rehabilitation Engineering, 6, 257-268. http://dx.doi.org/10.1109/86.712220

[26] Parsons, S., Mitchell, P. and Leonard, A. (2004) The Use and Understanding of Virtual Environments by Adolescents with Autistic Spectrum Disorders. Journal of Autism and Development Disorders, 34, 449-466. http://dx.doi.org/10.1023/B:JADD.0000037421.98517.8d

[27] Shane, H.C. and Albert, P.D. (2008) Electronic Screen Media for Persons with Autism Spectrum Disorders: Results of a Survey. Journal of Autism and Developmental Disorders, 38, 1499-1508. http://dx.doi.org/10.1007/s10803-007-0527-5

[28] Silver, M. and Oakes, P. (2001) Evaluation of a New Computer Intervention to Teach People with Autism or Asperger Syndrome to Recognise and Predict Emotions in Others. Autism, 5, 299-316. http://dx.doi.org/10.1177/1362361301005003007

[29] Hayes, N. (2000) Foundations of Psychology. Thomson Learning, London.

[30] Honey, E., McConachie, H., Turner, M. and Rodgers, J. (2012) Validation of the Repetitive Behaviour Questionnaire for Use with Children with Autism Spectrum Disorder. Research in Autism Spectrum Disorders, 6, 355-364. http://dx.doi.org/10.1016/j.rasd.2011.06.009

[31] Bodfish, J.W., Symons, F.J., Parker, D.E. and Lewis, M.H. (2000) Varieties of Repetitive Behaviour in Autism: Comparisons to Mental Retardation. Journal of Autism and Developmental Disorders, 30, 237-243. http://dx.doi.org/10.1023/A:1005596502855

[32] Newell, A. (1994) Unified Theories of Cognition. Harvard University Press, Cambridge, MA.

[33] Cheng, J., Greiner, R., Kelly, J., Bell, D. and Liu, W. (2002) Learning Bayesian Networks from Data: An Information-Theory Based Approach. Artificial Intelligence, 137, 43-90.

[34] Berry, D. and Broadbent, D. (1984) On the Relationship between Task Performance and Associated Verbalizable Knowledge. Quarterly Journal of Experimental Psychology, 36A, 209-231. http://dx.doi.org/10.1080/14640748408402156

[35] Nissen, M. and Bullemer, P. (1987) Attentional Requirements of Learning: Evidence from Performance Measures. Cognitive Psychology, 19, 1-32. http://dx.doi.org/10.1016/0010-0285(87)90002-8

[36] Willingham, D., Nissen, M. and Bullemer, P. (1989) On the Development of Procedural Knowledge. Journal of Experimental Psychology: Learning, Memory, and Cognition, 15, 1047-1060. http://dx.doi.org/10.1037/0278-7393.15.6.1047

[37] Lee, M.D. (2011) How Cognitive Modeling Can Benefit from Hierarchical Bayesian Models. Journal of Mathematical Psychology, 55, 1-7. http://dx.doi.org/10.1016/j.jmp.2010.08.013

[38] Orun, A.B. (2004) Automated Identification of Man-Made Textural Features on Satellite Imagery by Bayesian Networks. Photogrammetric Engineering and Remote Sensing, 70, 211-216. http://dx.doi.org/10.14358/PERS.70.2.211

[39] Orun, A.B. and Aydin, N. (2010) Variable Optimisation of Medical Image Data by the Learning Bayesian Network Reasoning. 32nd Annual International Conference of the IEEE Engineering in Medicine and Biology Society (EMBC'10), Buenos Aires, 1-4 September 2010, 4554-4557.

[40] Koski, T. and Noble, J. (2009) Bayesian Networks: An Introduction. John Wiley and Sons, Chichester. http://dx.doi.org/10.1002/9780470684023

[41] Pearl, J. (1988) Probabilistic Reasoning in Intelligence Systems: Network of Plausible Inference. Morgan Kaufmann, San Francisco.

[42] Touretzky, D., Mozer, M. and Hasselmo, M. (1996) Advances in Neural Information Processing Systems. Vol. 8, MIT Press, Cambridge, MA, 757-763.

[43] Mencap Organisation (2012). http://www.mencap.org.uk 